\title{
Dermatitis herpetiformis: a comparative assessment of skin and bowel abnormality
}

\author{
T. COONEY, C. T. DOYLE, D. BUCKLEY, AND M. J. WHELTON \\ From the Departments of Dermatology, Gastroenterology, and Pathology, St Finbarr's Hospital, Cork, \\ Ireland
}

SUMMARY We reviewed 18 patients with a clinical diagnosis of dermatitis herpetiformis who were being treated with dapsone and were on an unrestricted diet. Diagnosis was confirmed by finding IgA deposits in the dermal papillae of unaffected skin. Dapsone was discontinued and biopsy of affected skin was carried out when the typical rash reappeared. The biopsy findings were graded according to the severity of the histological changes. Small bowel tissue from each patient was examined and graded by stereo- and routine microscopy. Thirteen specimens $(72 \%)$ were stereomicroscopically abnormal; all 18 showed villous atrophy, either partial or subtotal; and in $13(72 \%)$ the interepithelial lymphocyte count was increased. No correlation was found between the histological severity of the skin and the small bowel lesions. Seemingly the severity of the skin rash in dermatitis herpetiformis is no guide to the degree of small bowel abnormality.

The occurrence of gluten-sensitive enteropathy in patients with dermatitis herpetiformis (DH) has been well established (Fry et al., 1967; Fry et al., 1968; Shuster et al., 1968; Fry et al., 1969; Fry et al., 1972). The incidence of small bowel mucosal lesions, however, varies widely in different series, ranging from $25 \%$ (Ruppin et al., 1975) to $81 \%$ (Scott et al., 1976), and to almost $100 \%$ (Fry et al., 1974). A patchy distribution has been suggested as a partial explanation of this variation in incidence (Brow et al., 1971; Scott and Losowsky, 1975). It has also been proposed (Fry et al., 1972; Stevens et al., 1975) that in some patients with DH the mucosal abnormality may be detected only by more sensitive techniques such as intestinal enzyme assay and interepithelial lymphocyte counting, and Fry et al. $(1972 ; 1974)$ have used the increased interepithelial lymphocyte count as an index of mucosal abnormality.

In studying the skin lesions of DH Piérard and Whimster (1961) laid down criteria which they considered characteristic of the disorder. Subsequent studies (Lever, 1965; Connor et al., 1972) suggested that these criteria, although characteristic of DH, were not in fact pathognomonic. Recent studies (Fry and Seah, 1974; Seah and Fry, 1975) propose that the finding of IgA in the dermal papillae of unaffected skin is the simplest and most reliable way of establishing the diagnosis.

In the light of these developments we reviewed 18

Received for publication 17 March 1977 clinically diagnosed, dapsone-treated patients with DH. Having confirmed the diagnosis by finding $\operatorname{IgA}$ in the dermal papillae of unaffected skin in all patients we withdrew dapsone and examined by biopsy both the affected skin (on the reappearance of the rash) and the small bowel in each patient. The purpose of the study was to see whether there was a correlation between the severity of the lesions in the skin and in the small bowel mucosa.

\section{Patients and methods}

Eighteen patients (11 men, 7 women) aged from 17 to 73 years were studied. Their DH had initially been diagnosed on the appearance, site, and pruritic nature of the rash together with its response to dapsone. They had been treated for periods varying from three months to 16 years and all were on a normal diet.

The period between withdrawal of dapsone and the appearance of the rash varied from four days to eight weeks. Tissue from maculopapular or vesicular skin lesions was taken for biopsy under local anaesthesia from the elbow region and processed for routine microscopy. Punch $(4 \mathrm{~mm})$ biopsy specimens of unaffected skin were taken under local anaesthesia from the extensor surface of the forearm distal to the elbow and at least $2 \mathrm{~cm}$ from the site of biopsy of affected skin. These specimens were snap frozen and examined by direct immunofluorescence using monospecific fluorescein isothiocyanate (FITC) conjugated anti-human IgG, IgM, and IgA. 
The histological features of the biopsies of affected skin were assessed according to the criteria of Piérard and Whimster (1961). The severity of the lesion in each case was graded according to the number of specific criteria present. Twelve criteria were used (Table 1).

Specimens for small bowel biopsy were taken from the proximal jejunum using a Crosby capsule or, when this was unsatisfactory, from the second or third part of the duodenum by direct vision using a side-viewing duodenoscope (JFB 2). The specimens were examined stereomicroscopically and graded into one of four categories: flat, convoluted, leaves only, and leaves and fingers. The specimens were then fixed in formol saline for routine processing and $5 \mu$ sections were stained with haematoxylin and eosin. The histological appearances were graded as normal, partial villous atrophy (mild, moderate, and severe), and flat (subtotal villous atrophy). Interepithelial lymphocyte counts were carried out on each specimen. In villous and convoluted specimens counts were made on the cells at the sides and tips of the villi and in flat specimens on the surface cells. Five hundred epithelial cells were counted in each specimen and the results were expressed as lymphocytes per 1000 epithelial cells. For the purpose of this study we accepted as abnormal counts of over 200 lymphocytes per 1000 epithelial cells (Fry et al., 1974). Further reference will be made to this point in the discussion.

\section{Results}

The number of histological criteria fulfilled in assessing the biopsies of affected skin is shown in Table 2. In nine patients $(50 \%)$ not more than eight criteria were fulfilled. Only two patients $(11 \%)$ fulfilled all 12 criteria. Fibrillary or microgranular IgA

Table 1 Skin biopsy criteria in dermatitis herpetiformis (Piérard and Whimster, 1961)

\begin{tabular}{ll}
\hline Site & Specific features \\
\hline Papillary dermis & $\begin{array}{l}\text { Oedema of papillae } \\
\text { Papillary microabscesses } \\
\text { Connective tissue necrosis }\end{array}$ \\
Floor of lesion & $\begin{array}{l}\text { Papillary outlines } \\
\text { Polymorphs and eosinophils } \\
\text { Pyknotic nuclei and cellular debris }\end{array}$ \\
Fluid within blister & $\begin{array}{l}\text { Fibrin threads } \\
\text { Polymorphs and eosinophils } \\
\text { Shadow epidermal cells } \\
\text { Roof of lesion }\end{array}$ \\
& $\begin{array}{l}\text { Stretching of epidermis } \\
\text { Progressive necrosis of lower epidermal layers } \\
\text { Epidermis cleanly detached }\end{array}$ \\
\hline
\end{tabular}

Table 2 Histological assessment of biopsies of small bowel and affected skin in 18 patients with dermatitis herpetiformis

\begin{tabular}{|c|c|c|c|c|}
\hline $\begin{array}{l}\text { Case } \\
\text { no. }\end{array}$ & Stereomicroscopy & $\begin{array}{l}\text { Routine } \\
\text { microscopy }\end{array}$ & $\begin{array}{l}\text { Interepithelial } \\
\text { lymphocytes }\end{array}$ & $\begin{array}{l}\text { No. of } \\
\text { criteria } \\
\text { fulfilled } \dagger\end{array}$ \\
\hline 1 & Leaves & PVA, moderate & 205 & 3 \\
\hline 2 & Convoluted & PVA, severe & 400 & 5 \\
\hline 3 & Flat & Flat & 296 & 8 \\
\hline 4 & Flat & Flat & 327 & 10 \\
\hline 5 & $\begin{array}{l}\text { Leaves and } \\
\text { fingers }\end{array}$ & PVA, mild & 155 & 10 \\
\hline 6 & $\begin{array}{l}\text { Leaves and } \\
\text { fingers }\end{array}$ & PVA, severe & 420 & 10 \\
\hline 7 & Convoluted & PVA, severe & 184 & 11 \\
\hline 8 & Convoluted & PVA, severe & 397 & 12 \\
\hline 9 & Convoluted & PVA, severe & 263 & 1 \\
\hline 10 & Flat & Flat & 390 & 4 \\
\hline 11 & Convoluted & PVA, moderate & 240 & 10 \\
\hline 12 & Flat & Flat & 180 & 12 \\
\hline 13 & Convoluted & PVA, moderate & 265 & 8 \\
\hline 14 & Convoluted & PVA, moderate & 137 & 11 \\
\hline 15 & Convoluted & PVA, severe & 316 & 10 \\
\hline 16 & Convoluted & PVA, severe & 328 & 7 \\
\hline 17 & Leaves & PVA, moderate & 247 & 4 \\
\hline 18 & Leaves & PVA, mild & 180 & 4 \\
\hline
\end{tabular}

PVA $=$ partial villous atrophy.

*Interepithelial lymphocytes/1000 epithelial cells.

† Maximum no. possible $=12$.

deposits, however, were detected in the papillary dermis of unaffected skin in all patients.

The appearance of the small bowel biopsies is also listed in Table 2. The steromicroscopic appearance was abnormal in 13 cases (72\%): four were flat and nine convoluted. Five specimens $(28 \%)$ were stereomicroscopically normal: three showed leaves and two leaves and fingers. Routine histology showed all 18 specimens to be abnormal: four $(22 \%)$ were flat and $14(78 \%)$ showed partial villous atrophy of varying degree. The interepithelial lymphocyte count was raised in 13 cases $(72 \%)$ : in seven $(38 \%)$ it was greater than 300 per 1000 epithelial cells; in six (33\%) between 200 and 300 ; and in five (28\%) below 200 . Comparison of the relative severity of skin and bowel lesions in each patient failed to show any correlation (Figs 1-3).

\section{Discussion}

The patients studied were originally diagnosed on the clinical appearance, distribution and pruritic nature of the rash, and the response to dapsone. Fry and Seah (1974) and Seah and Fry (1975) thought that the finding of IgA in the dermal papillae of the unaffected skin was the most specific diagnostic criterion of DH. We have taken this finding to be highly suggestive of $\mathrm{DH}$. All our patients had this abnormality, whereas the histological changes on routine microscopy showed a wide variation. As with the study of Fry and Seah (1974), histological examination of 


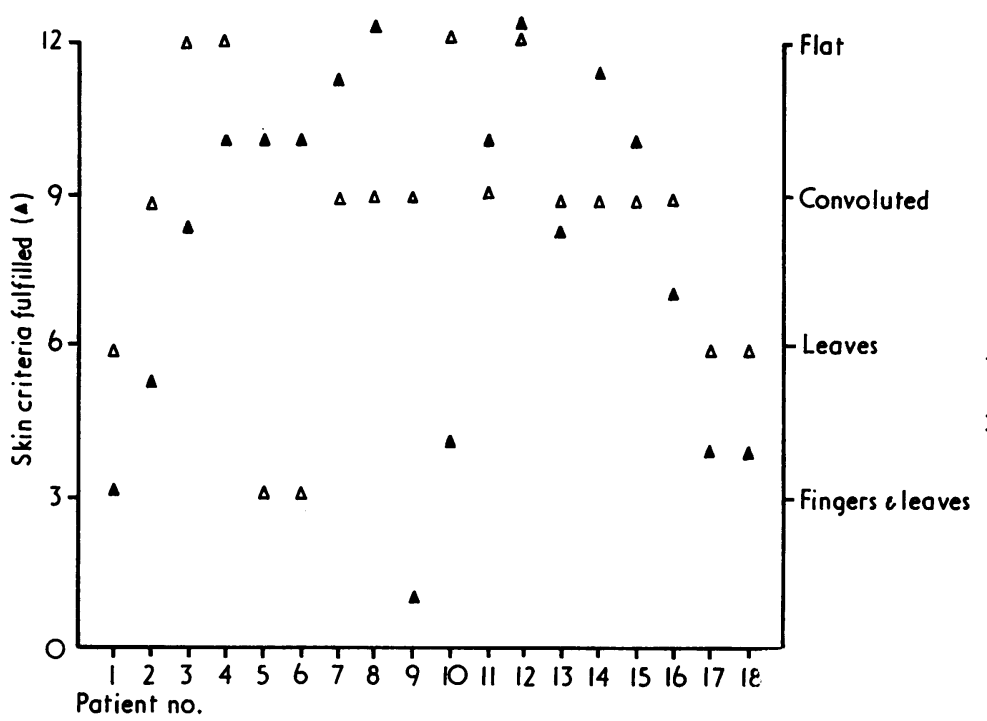

Fig. 1 Graphical representation of skin criteria fulfilled $(\mathbf{\Delta})$ when compared with stereomicroscopic appearance of bowel $(\triangle)$ in each patient. No correlation seen.

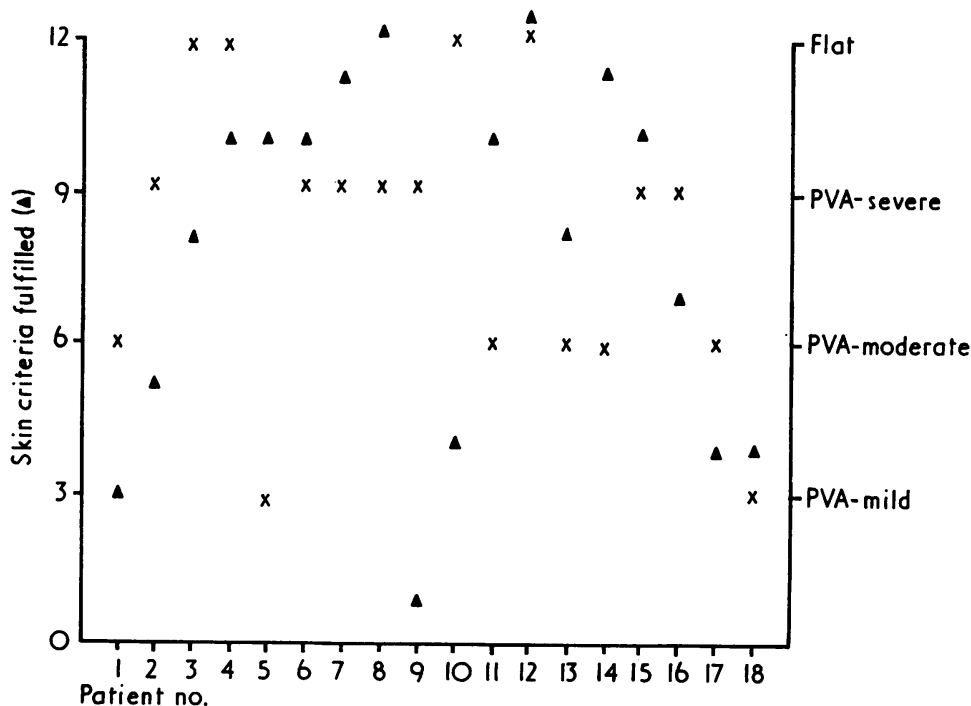

Fig. 2 Graphical representation of skin criteria fulfilled $(\mathbf{\Lambda})$ when compared with routine microscopic appearance of bowel $(\mathrm{X})$ in each patient. No correlation seen.

affected skin was altogether unhelpful in establishing a diagnosis in six patients $(33 \%)$ whose biopsies showed five or fewer of the requisite criteria. The criteria of Piérard and Whimster (1961), although characteristic, are not pathognomonic of DH. Nevertheless, they remain a useful basis on which to grade the severity of skin lesions in this disorder. The finding of IgA in the dermal papillae of unaffected skin, if shown conclusively to be specific for $\mathrm{DH}$, would be a valuable diagnostic tool.

All our patients had evidence of enteropathy. Histological abnormalities were noted in all (Table 2); $13(72 \%)$ were abnormal on stereomicroscopy; and interepithelial lymphocyte counts were raised in $13(72 \%)$. There was reasonably good correlation between the degree of villous atrophy on the one hand and both the stereomicroscopic appearance and the $\triangle$ interepithelial lymphocyte count on the other. The 
$\circ$

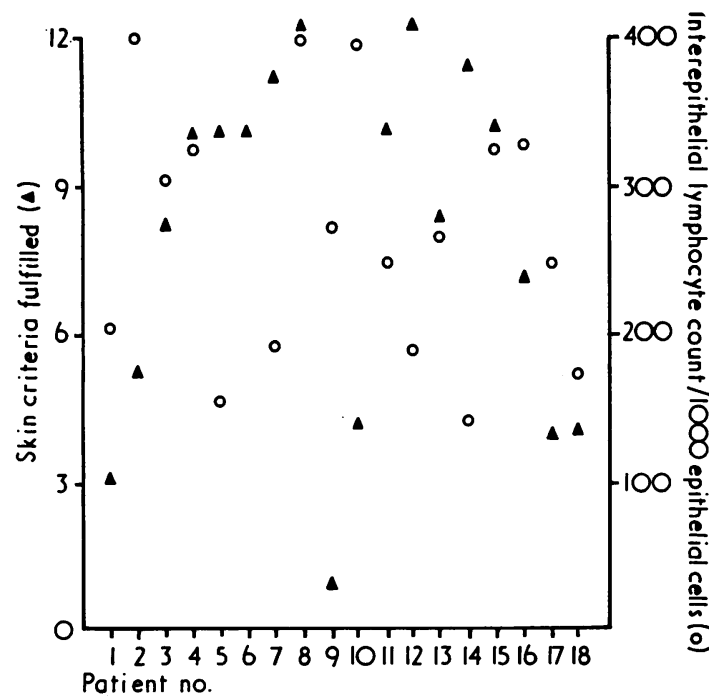

Fig. 3 Graphical representation of skin criteria fulfilled $(\Delta)$ when compared with interepithelial lymphocyte count $(0)$ in each patient. No correlation seen.

consistently abnormal small bowel histology in the present series contrasts with the findings of Scott $e t$ al. (1976), who reported $23 \%$ of patients in whom biopsy was normal on both stereomicroscopy and routine histology. This discrepancy may be partly explained by the apparently higher incidence of coeliac disease in Ireland (Mylotte et al., 1973). Equally it may be argued that our cases 2 and 18 (Table 2) with normal stereomicroscopic appearances, normal interepithelial lymphocyte counts, and only mild partial villous atrophy are in fact variations of normal. We think, however, bearing in mind the patchy distribution of the small bowel lesions in DH (Brow et al., 1971; Scott and Losowsky, 1975), that the finding of even a mild degree of villous atrophy in a single biopsy in these patients is significant. The incidence of mucosal abnormality in our series accords best with that of Fry et al. (1974).

The exact definition of a raised interepithelial lymphocyte count and the precise significance of such a finding remain to be fully elucidated. Several studies have proposed a normal range for interepithelial lymphocytes, but these normals vary per 1000 epithelial cells from 69-285 (Fry et al., 1974), 80-270 (Mavromichalis et al., 1976), 60-400 (Ferguson and Murray, 1971), to 74-428 (Scott et al., 1976), and 132-450 (Stevens et al., 1975). We have applied the range of Fry et al. (1974) (69-285), in which all but four of their controls had counts of under 200 , since this accorded best with the stereo- and routine microscopic findings in our patients. The nonspecificity of a raised interepithelial lymphocyte count as shown by Mavromichalis et al. (1976) indicates the need for correlating the count with both the microscopic appearance of the mucosa and the response to gluten withdrawal and challenge if a diagnosis of coeliac disease is to be made. We have also noted that several biopsies showing morphologically abnormal villi had normal interepithelial lymphocyte counts, although Mavromichalis et al. (1976) found this phenomenon only in enteropathies other than coeliac disease. The range of interepithelial lymphocytes in our patients (137-420) is considerably lower than that reported in abnormals in similar series. The reason for this is not clear.

The purpose of this study was to see whether there is a correlation between the severity of the skin lesions and those of the small bowel in DH. Evidently (Figs. 1-3) there is no such correlation. Thus in all patients with DH biopsy of the small bowel should be done to ascertain the degree of mucosal abnormality irrespective of the state of the skin. Diagnosis of villous atrophy in these patients merits treatment with a gluten free diet both to correct the intestinal abnormality (Fry et al., 1968; Marks and Whittle, 1969; Weinstein et al., 1970) and to minimise the dose of drugs necessary to control the skin lesions (Heading et al., 1976). A gluten free diet may not prevent malignant complications in patients with coeliac disease (Holmes et al., 1976). The evidence for this is as yet by no means conclusive, and until it is we think that a gluten free diet for patients with severe coeliac disease remains mandatory simply because it may prevent malignancy.

We are indebted to D. A. McQueen, Department of Dermatology, University of Glasgow, for the skin immunofluorescence studies.

\section{References}

Brow, J. R., Parker, F., Weinstein, W. M., and Rubin, C. E. (1971). The small intestinal mucosa in dermatitis herpetiformis. I. Severity and distribution of the small intestinal lesion and associated malabsorption. Gastroenterology, 60, 355-361.

Connor, B. L., Marks, R., and Jones, E. W. (1972). Dermatitis herpetiformis: histological discriminants. Transactions of the St. John's Hospital Dermatological Society, 58, 191-198.

Ferguson, A., and Murray, D. (1971). Quantitation of intraepithelial lymphocytes in human jejunum. Gut, 12, 988-994.

Fry, L., Keir, P., McMinn, R. M. H., Cowan, J. D., and Hoffbrand, A. V. (1967). Small-intestinal structure and function and haematological changes in dermatitis herpetiformis. Lancet, 2, 729-734. 
Fry, L., McMinn, R. M. H., Cowan, J. D., and Hoffbrand, A. V. (1968). Effect of gluten-free diet on dermatological, intestinal, and haematological manifestations of dermatitis herpetiformis. Lancet, 1, 557-561.

Fry, L., McMinn, R. M. H., Cowan, J. D., and Hoffbrand, A. V. (1969). Gluten-free diet and reintroduction of gluten in dermatitis herpetiformis. Archives of Dermatology, 100, 129-135.

Fry, L., and Seah, P. P. (1974). Dermatitis herpetiformis: an evaluation of diagnostic criteria. British Journal of Dermatology, 90, 137-146.

Fry, L., Seah, P. P., Harper, P. G., Hoffbrand, A. V., and McMinn, R. M. H. (1974). The small intestine in dermatitis herpetiformis. Journal of Clinical Pathology, 27, 817-824.

Fry, L. Seah, P. P., McMinn, R. M. H., and Hoffbrand, A. V. (1972). Lymphocytic infiltration of epithelium in diagnosis of gluten-sensitive enteropathy. British Medical Journal, 3, 371-374.

Heading, R. C., Paterson, W. D., McClelland, D. B. L., Barnetson, R. St. C., and Murray, M. S. M. (1976). Clinical response of dermatitis herpetiformis skin lesions to a gluten-free diet. British Journal of Dermatology, 94, 509-514.

Holmes, G. K. T., Stokes, P. L., Sorahan, T. M., Prior, P., Waterhouse, J. A. H., and Cooke, W. T. (1976). Coeliac disease, gluten-free diet, and malignancy. Gut, 17, 612-619.

Lever, W. F. (1965). Pemphigus and Pemphigoid. Thomas, Springfield, Illinois.

Marks, R., and Whittle, M. W. (1969). Results of treatment of dermatitis herpetiformis with a gluten-free diet after one year. British Medical Journal, 4, 772-775.

Mavromichalis, J., Brueton, M. J., McNeish, A. S., and Anderson, C. M. (1976). Evaluation of the intraepithelial lymphocyte count in the jejunum in childhood enteropathies. Gut, 17, 600-603.

Mylotte, M., Egan-Mitchell, B., McCarthy, C. F., and McNicholl, B. (1973). The incidence of coeliac disease in the West of Ireland. British Medical Journal, 1, 703705.

Piérard, J., and Whimster, I. (1961). The histological diagnosis of dermatitis herpetiformis, bullous pemphigoid and erythema multiforme. British Journal of Dermatology, 73, 253-266.

Ruppin, H., Weidner, F., Domschke, S., Domschke, W., Classen, M., and Hornstein, O. P. (1975). Dermatitis herpetiformis and small intestinal lesion-no strict association in German patients. Acta Hepato-Gastroenterologica, 22, 105-111.

Scott, B. B., and Losowsky, M. S. (1975). Patchiness of the mucosal abnormality in coeliac disease (CD) and dermatitis herpetiformis (DH). Gut, 16, 393. (Abstract).

Scott, B. B., Young, S., Rajah, S. M., Marks, J., and Losowsky, M. S. (1976). Coeliac disease and dermatitis herpetiformis: further studies of their relationship. Gut, 17, 759-762.

Seah, P. P., and Fry, L. (1975). Immunoglobulins in the skin in dermatitis herpetiformis and their relevance in diagnosis. British Journal of Dermatology, 92, 157-166.

Shuster, S., Watson, A. J., and Marks, J. (1968). Coeliac syndrome in dermatitis herpetiformis. Lancet, 1, 11011106.

Stevens, F. M., Lloyd, R., Egan-Mitchell, B., Mylotte, M. J., Fottrell, P. F., Wright, R., McNicholl, B., and McCarthy, C. F. (1975). Reticulin antibodies in patients with coeliac disease and their relatives. Gut, 16, 598602.

Weinstein, W. M., Brow, J. R., Parker, F., and Rubin, C. E. (1970). The small intestinal mucosa in dermatitis herpetiformis. 2. Relationship of the small intestinal lesion to gluten. Gastroenterology, 60, 362-369. 